\title{
Backward Stochastic Differential Equations Approach to Hedging, Option Pricing, and Insurance Problems
}

\author{
Francesco Cordoni ${ }^{1}$ and Luca Di Persio ${ }^{2}$ \\ ${ }^{1}$ Department of Mathematics, University of Trento, Via Sommarive 14, 38123 Trento, Italy \\ ${ }^{2}$ Department of Computer Science, University of Verona, Strada le Grazie 15, 37134 Verona, Italy
}

Correspondence should be addressed to Luca Di Persio; dipersioluca@gmail.com

Received 30 May 2014; Accepted 25 August 2014; Published 11 September 2014

Academic Editor: Ciprian A. Tudor

Copyright ( 2014 F. Cordoni and L. Di Persio. This is an open access article distributed under the Creative Commons Attribution License, which permits unrestricted use, distribution, and reproduction in any medium, provided the original work is properly cited.

In the present work we give a self-contained introduction to financial mathematical models characterized by noise of Lévy type in the framework of the backward stochastic differential equations theory. Such techniques will be then used to analyse an innovative model related to insurance and death processes setting.

\section{Introduction}

The main goal of the work consists in the analysis of problems characterizing modern financial markets which can be efficiently studied using techniques coming from the theory of stochastic differential equations in backward form (BSDEs); see, for example, [1-3]. We start giving an overview of classical approaches, for example, the standard BlackScholes (B-S) model, Section 3.1, which are related to the use of stochastic perturbations of Brownian type. Then we will enrich the description of the relevant stock's dynamics by considering first stochastic jumps, Section 3.3, and after full Lévy type noises, also including related Girsanov theorem and Esscher transform, Section 2.2. Such an analysis will allow us, Section 3.4, to study some original applications in particular with respect to life insurance's problems and linked death processes.

A key ingredient of our analysis will be an extensive use of the theory of backward stochastic differential equations (BSDEs) introduced by Bismut (1973), for the linear case, and generalized by Pardoux and Peng (1990) in the general nonlinear case in the Brownian framework; see, for example [4].

In [5] Pardoux and Peng provided also a FeynmanKac type theorem for solution of nonlinear parabolic partial differential equation (PDE). We would like to underline that
BSDEs techinques provide powerful instruments to analyse a heterogeneous class of concrete problems, spanning from biology to finance, from population dynamics to particle theory, and so forth; see, for example, [2, 3, 6, 7] and references therein.

In the mathematical finance framework BSDEs techniques gained a great attention by both practitioners and academics in particular with respect to problems which arise in option pricing, portfolio hedging, market utility maximization, risk measures, and so forth. Latter interests have been increasingly developed both in financial as well in insurance frameworks, particularly under the influence of the related European directives, namely, Basilea and Solvency; see, for example, [3] for one of the first review on the subject and $[1,2]$ for a more extensive introduction to recent developments.

First approaches to such kind of general quantitative economic questions have been following the idea of Black and Scholes, hence developed in a Brownian setting, namely, allowing the dynamics of the interested quantities to be driven by Brownian measure/noise. Nevertheless empirical evidence, for example, working with stock prices' behaviours, has pointed out that the traditional setup was based on the geometric Brownian motion; it is not fully satisfactory since it lacks an accurate description of financial data; see, for example, $[8,9]$. A typical example of such type of issues arise 
in the study of the implied volatility surface; see, for example, $[8,10]$.

Moreover, by its own nature, Brownian models are not able to capture those phenomena which are characterized by abrupt changes in economic quantities of interest, changes which have become more and more frequently determining in current financial fields.

Latter issues have promoted the development of more flexible models causing an explosion of the related mathematical literature starting from the late 90s; see, for example, $[1,2,11,12]$ and references therein.

Following these impulses great improvements towards more realistic models to describe and forecast movements of relevant financial quantities have been achieved taking into account Lévy type noises, hence allowing for asset price dynamics influenced by random perturbation with both diffusive and jump components. The Lévy random jump part is a key ingredient to capture sudden variations of prices which could happen, for example, in presence of turbulent economics dynamics originated by unexpected political events, natural disasters, abrupt variations of commodities' prices, and so forth.

Fundamental results related to BSDEs in presence of Lévy type drivers are given by Rong in [13], where BSDEs driven by a Brownian motion plus a Poisson point process are studied, and in [14], where Ouknine exploited the integral representation of a square-integrable random variable in terms of a Poisson random, to study the case of a BSDEs driven by a Poisson random measure.

Moreover, in [15], Nualart and Schoutens proved both a martingale representation theorem for Lévy processes satisfying some exponential moment condition and a Feynman-Kac formula using a Teugels type orthonormalization procedure.

In what follows, we will consider the wealth processes dynamic of a portfolio, composed by a riskless asset, for example, a bond or a bank account, and a risky security whose dynamic is modelled using a BSDE driven by a Lévy process, hence generalizing the classic approach based on Brownian stochastic driver.

In particular the paper is organized as follows. In Section 2 basic definitions for the mathematical framework within to develop BSDEs' theory characterized by Lévy type noise are stated and the role played by both the Girsanov theorem and the Esscher transform is underlined with applications in view. In Section 3 the financial background will be set up and we will provide related examples; see Sections 3.1, 3.2, and 3.3; moreover in Section 3.4 a novel insurance application is presented.

\section{Mathematical Framework}

In this section we will give basic definitions and results which allow to set up a suitable framework within hedging/pricing problems which will be analysed in Section 3.

Let $W_{t}=\left\{W_{t}\right\}_{t \in[0, T]}, T \in \mathbb{R}^{+}$, be a one dimensional Brownian motion on a probability space $(\Omega, \mathscr{F}, \mathbb{P})$ and let $N(d x, d t)$ be a Poisson random measure on $\mathbb{R}^{+} \times \mathbb{R}^{*}$, where we have denoted $\mathbb{R}^{*}:=\mathbb{R} \backslash\{0\}$, independent of $W_{t}$, with compensator $v(d x) d t$ and such that it is a $\sigma$-finite Lévy measure on $\left(\mathbb{R}^{*}, \mathscr{B}\left(\mathbb{R}^{*}\right)\right)$, with $\widetilde{N}(d t, d u):=N(d x, d t)-v(d x) d t$ representing its compensated measure. Let $\left(\mathscr{F}_{t}\right)_{t \in[0, T]}$ be the filtration generated (jointly) by $W_{t}$ and $N_{t}$, while $\mathscr{P}$ is the predictable $\sigma$-algebra on $[0, T] \times \Omega$. Throughout the paper the following notations will be used.

(i) $L^{2}\left(\mathscr{F}_{T}\right)$ is the set of random variables which are $\mathscr{F}_{T}$ measurable and square integrable:

$$
L^{2}\left(\mathscr{F}_{T}\right):=\left\{Z: \Omega \longrightarrow \mathbb{R}: \int_{\Omega}|Z(\omega)|^{2} \mathbb{P}(d \omega)<\infty\right\},
$$

endowed with the scalar product

$$
\langle Y, Z\rangle:=\int_{\Omega} Y(\omega) Z(\omega) \mathbb{P}(d \omega)
$$

(ii) $\mathscr{H}^{2, T}$ is the set of real-valued progressively measurable processes:

$$
\begin{aligned}
\mathscr{H}^{2, T}:=\{X & =\left\{X_{t}: 0 \leq t \leq T\right\}: \\
& \left.\mathbb{E}\left[\int_{0}^{T}\left|X_{t}(\omega)\right|^{2} d t\right]<\infty\right\},
\end{aligned}
$$

endowed with the scalar product

$$
\left\langle X^{1}, X^{2}\right\rangle_{\mathscr{H}}:=\mathbb{E}\left[\int_{0}^{T} X_{t}^{1}(\omega) X_{t}^{2}(\omega) d t\right]
$$

(iii) $L^{2}\left(\mathbb{R}^{*}, v\right)$ is the set of square $v$-measurable functions

$$
L^{2}\left(\mathbb{R}^{*}, \nu\right):=\left\{\gamma: \mathbb{R}^{*} \longrightarrow \mathbb{R}: \int_{\mathbb{R}^{*}}|\gamma(x)|^{2} \nu(d x)<\infty\right\},
$$

endowed with the scalar product

$$
\langle\gamma, \eta\rangle_{\nu}:=\int_{\mathbb{R}^{*}} \gamma(x) \eta(x) \nu(d x)
$$

(iv) $\mathscr{H}_{v}^{2, T}$ is the set of predictable progressively measurable stochastic processes

$$
\begin{gathered}
\mathscr{H}_{\nu}^{2, T}:=\left\{\gamma=\left\{\gamma_{t}(\omega, x): 0 \leq t \leq T, \omega \in \Omega, x \in \mathbb{R}^{*}\right\}:\right. \\
\left.\mathbb{E}\left[\int_{0}^{T} \int_{\mathbb{R}^{*}}\left|\gamma_{t}(\omega, x)\right|^{2} \nu(d x) d t\right]<\infty\right\},
\end{gathered}
$$

endowed with the scalar product

$$
\left\langle\gamma^{1}, \gamma^{2}\right\rangle_{\mathscr{H}}:=\mathbb{E}\left[\int_{0}^{T} \int_{\mathbb{R}^{*}} \gamma_{t}^{1}(\omega, x) \gamma_{t}^{2}(\omega, x) \nu(d x) d t\right]
$$


(v) $S^{2, T}$ is the set of real-valued cádlág adapted processes

$$
\begin{aligned}
& S^{2, T}:=\{X=\left\{X_{t}(\omega): 0 \leq t \leq T\right\}: \\
&\left.\mathbb{E}\left[\sup _{0 \leq t \leq T}\left|X_{t}(\omega)\right|^{2}\right]<\infty\right\},
\end{aligned}
$$

endowed with the norm

$$
\|X\|_{S}:=\mathbb{E}\left[\sup _{0 \leq t \leq T}\left|X_{t}(\omega)\right|^{2}\right] ;
$$

(vi) $\tau_{0}$ is the set of stopping times $\tau$, such that $\tau \in[0, T]$, a.s.

Moreover we will use the following simplified notations: $L^{2}:=L^{2}\left(\mathscr{F}_{T}\right), \mathscr{H}:=\mathscr{H}^{2, T}, L_{v}^{2}:=L^{2}\left(\mathbb{R}^{*}, \nu\right)$, and $\mathscr{H}_{\nu}:=\mathscr{H}_{\nu}^{2, T}$, which are Hilbert spaces, with the respective scalar product, whenever it makes sense. Furthermore we set $S:=S^{2, T}$ and we will omit the explicit dependence on $\omega$ whenever it does not cause misinterpretations.

2.1. Linear BSDE. In what follows we introduce some fundamental results in the theory BSDEs; see, for example, [2,3], for a deeper treatment on the topic. We start studying the cases of linear BSDEs for which a solution can be expressed as a conditional expectation of some specified known processes; later, in Section 3, explicit formulae for such solutions will be provided. Taking into account previous notations, we define a BSDE with jumps as follows.

Definition 1 (BSDE). A BSDE with jumps is an equation of the form

$$
\begin{gathered}
-d X_{t}=f\left(t, X_{t^{-}}, Y_{t}, U_{t}\right) d t-Y_{t} d W_{t} \\
-\int_{\mathbb{R}^{*}} U_{t}(x) \widetilde{N}(d t, d x), \\
X_{T}=\xi,
\end{gathered}
$$

where $W_{t}$ is a one-dimensional Brownian motion, $\widetilde{N}(d t, d x)$ is the compensated Poisson random measure on $\mathbb{R}^{+} \times \mathbb{R}^{*}$ defined above, and $\xi$ is the so-called terminal condition.

Definition 2 (driver). A function $f:[0, T] \times \Omega \times \mathbb{R} \times \mathbb{R} \times L_{v}^{2} \rightarrow$ $\mathbb{R}$ is called a driver for the BSDE (11), if it holds the following properties:

(i) $f$ is $\mathscr{P} \otimes \mathscr{B}\left(\mathbb{R}^{2}\right) \otimes \mathscr{B}\left(L_{v}^{2}\right)$-measurable,

(ii) $f(\cdot, 0,0,0) \in \mathscr{H}$.

Definition 3 (Lipschitz driver). Let $f$ be a driver of a BSDE of the form (11) in the sense of Definition 2; then $f$ is said to be a Lipschitz driver if there exists a constant $C \geq 0$ such that, for each $\left(x_{1}, y_{1}, u_{1}\right),\left(x_{2}, y_{2}, u_{2}\right) \in \mathbb{R} \times \mathbb{R} \times L_{v}^{2}$, the following holds:

$$
\begin{gathered}
\left|f\left(t, w, x_{1}, y_{1}, u_{1}\right)-f\left(t, w, x_{2}, y_{2}, u_{2}\right)\right| \\
\leq C\left(\left|x_{1}-x_{2}\right|+\left|y_{1}-y_{2}\right|\right. \\
\left.\quad+\left|u_{1}-u_{2}\right|_{v}\right), \quad d \mathbb{P} \otimes d t \text { a.s. }
\end{gathered}
$$

Definition 4. A solution to a BSDE is a triplet $\left(X_{t}, Y_{t}, U_{t}\right) \in$ $S \times \mathscr{H} \times \mathscr{H}_{\nu}$ satisfying

$$
\begin{aligned}
X_{t}= & \xi+\int_{t}^{T} f\left(t, X_{s^{-}}, Y_{s}, U_{s}\right) d s-\int_{t}^{T} Y_{s} d W_{s} \\
& -\int_{t}^{T} \int_{\mathbb{R}^{*}} U_{s}(x) \widetilde{N}(d s, d x),
\end{aligned}
$$

where $X_{t}$ is a cádlág process, $Y_{t}$ is progressively measurable, and $U_{t}$ is integrable with respect to the compensated Poisson measure $\widetilde{N}(d t, d x)$.

Existence and uniqueness results for the problem (11) can be established provided that suitable conditions on the terminal condition $\xi$ and the driver $f$ are satisfied; see, for example, [16] and references therein for details. In particular the following theorem holds.

Theorem 5. Let $f$ be as in Definition 3 and $\xi \in L^{2}$; then there exists a unique solution $\left(X_{t}, Y_{t}, U_{t}\right) \in S \times \mathscr{H} \times \mathscr{H}_{\nu}$ of (11).

With financial application in mind we will consider, as a special case of the previous result, BSDEs with linear generator, hence taking $f$ of the form

$$
f\left(t, X_{t}, Y_{t}, U_{t}\right)=\alpha_{t}+\beta_{t} X_{t}+\delta_{t} Y_{t}+\left\langle U_{t}, \zeta_{t}\right\rangle_{v},
$$

where $\beta_{t}$ and $\delta_{t}$ are a real-valued predictable processes, supposed to be a.s. integrable with respect to $d t$ and $d W_{t}$, $\left(\zeta_{t}(\cdot)\right)_{t \in[0, T]}$ is a real-valued predictable process defined on $[0, T] \times \Omega \times \mathbb{R}^{*}$, that is, $\mathscr{P} \otimes \mathscr{B}\left(\mathbb{R}^{*}\right)$-measurable, and integrable with respect to $\widetilde{N}(d t, d x)$ and $\alpha_{t} \in \mathscr{H}$.

The following fundamental result shows that the solution of a linear BSDE with jumps can be written as a conditional expectation via an exponential semimartingale.

Theorem 6. Let $\left(X_{t}, Y_{t}, U_{t}\right)$ be the solution in $S \times \mathscr{H} \times \mathscr{H}_{\nu}$ of the following linear $B S D E$ :

$$
\begin{gathered}
-d X_{t}=\left(\alpha_{t}+\beta_{t} X_{t}+\delta_{t} Y_{t}+\left\langle U_{t}, \zeta_{t}\right\rangle_{v}\right) d t-Y_{t} d W_{t} \\
-\int_{\mathbb{R}^{*}} U_{t}(x) \widetilde{N}(d t, d x), \\
X_{T}=\xi ;
\end{gathered}
$$

then, the process $X_{t}$ satisfies

$$
X_{t}=\mathbb{E}\left[S_{T} \xi+\int_{t}^{T} S_{s} \alpha_{s} d s \mid \mathscr{F}_{t}\right], \quad 0 \leq t \leq T \text { a.s. }
$$


where the process $S_{t} \in S$ satisfies

$$
\begin{gathered}
d S_{t}=S_{t_{-}}\left(\beta_{t} d t+\delta_{t} d W_{t}+\int_{\mathbb{R}^{*}} \zeta_{t}(x) \widetilde{N}(d t, d x)\right) \\
S_{0}=1 .
\end{gathered}
$$

Proof. See, for example, [17].

2.2. Girsanov Theorem and the Change of Measure. In this section we focus our attention on the Girsanov theorem, a result which will be later used, Section 3, to obtain pricing relation by a change of measure approach when the asset prices' behaviour is represented by geometric Itô-Lévy processes, namely, by an analogue of the risk-neutral valuation technique used in the Black-Scholes framework. We would like to underline that (see, for example, $[3,8,18-20]$ ) when assets' dynamics are driven by jump-diffusion processes perfect hedge does not exist; namely, it is not always possible to replicate the derivative payoff by a controlled portfolio of the basic securities. However there exist particular cases, for example, when the driving process is of geometric Itô-Lévy type, which can be successfully treated by mean of the socalled Esscher transformation which allow for the definition of a suitable risk-neutral density in the form of the DoléansDade exponential; see below for details. In particular the following particular case for the Girsanov theorem holds; see, for example, $[8,18,21]$.

Theorem 7 (Girsanov theorem). Let $\left(\Omega, \mathscr{F},\left(\mathscr{F}_{t}\right)_{t \in[0, T]}, \mathbb{P}\right)$, where $\left(\mathscr{F}_{t}\right)_{t \in[0, T]}$ is the filtration generated (jointly) by $W_{t}$ and $N_{t}$, be a filtered probability space and let $S_{t}$ be a Geometric ItôLévy process of the form

$$
d S_{t}=S_{t^{-}}\left[\mu_{t} d t+\sigma_{t} d W_{t}+\int_{\mathbb{R}^{*}} \gamma_{t}(x) \widetilde{N}(d t, d x)\right]
$$

Let one further assume that, for any $\alpha \in \mathbb{R}$, the following holds:

$$
\int_{|x| \geq 1} e^{\alpha x} v(d x)<\infty
$$

and let $u_{t}, l_{t}(x)$ be two $\mathscr{F}_{t}$-predictable processes such that

(i)

$$
\mu_{t}+u_{t} \sigma_{t}+\int_{\mathbb{R}^{*}} \gamma_{t}(x) l_{t}(x) \nu(d x)=0
$$

(ii) the process $l_{t}(x)$ satisfies $l_{t}(x) \geq 0$,

(iii) the process $Z_{t}$ defined by the solution of the following SDE:

$$
\begin{gathered}
d Z_{t}=Z_{t^{-}}\left[u_{t} d W_{t}+\int_{\mathbb{R}^{*}} l_{t}(x) \widetilde{N}(d t, d x)\right]=Z_{t^{-}} d Y_{t} \\
Z_{0}=1
\end{gathered}
$$

where the process $Y_{t}$ satisfies

$$
d Y_{t}=u_{t} d W_{t}+\int_{\mathbb{R}^{*}} l_{t}(x) \widetilde{N}(d t, d x),
$$

is well defined and satisfies

$$
\mathbb{E}\left[Z_{T}\right]=1 \text {. }
$$

Then there exists a probability measure $\mathbb{Q}$ on $\mathscr{F}_{T}$ which is equivalent to $\mathbb{P}$ and such that

$$
d \mathbb{Q}=Z_{T} d \mathbb{P} .
$$

Note that in financial applications the Girsanov theorem is particularly useful since it allows the discounted stock price $e^{-r t} S_{t}$, where $r$ is the risk-free interest rate and $S_{t}$ is the stock price, to be a local martingale with respect to the measure $\mathbb{Q}$. Theorem 7 straightforwardly implies the following result; see, for example, [22].

Corollary 8. Let $u_{t}$ and $l_{t}(x) \geq 0$ be predictable processes such that the process $Z_{t}$ satisfying

$$
d Z_{t}=Z_{t^{-}}\left[u_{t} d W_{t}+\int_{\mathbb{R}^{*}} l_{t}(x) \widetilde{N}(d t, d x)\right],
$$

is well defined for $0 \leq t \leq T$. Suppose that

$$
\mathbb{E}\left[Z_{T}\right]=1,
$$

and define the probability measure $\mathbb{Q}$ on $\mathscr{F}_{T}$ by

$$
d \mathbb{Q}=Z_{T} d \mathbb{P}
$$

then

(i) the process $W_{t}^{\mathbb{Q}}$ defined by

$$
W_{t}^{\mathbb{Q}}=W_{t}-\int_{0}^{t} u_{s} d s,
$$

is a $\mathbb{Q}$-Brownian motion and

(ii) the random measure $\widetilde{N}^{\mathbb{Q}}(d t, d x)$ defined by

$$
\widetilde{N}^{\mathbb{Q}}(d t, d x)=\widetilde{N}(d t, d x)-l_{t}(x) v(d x) d t,
$$

such that

$$
\begin{aligned}
& \int_{0}^{t} \int_{\mathbb{R}^{*}} \widetilde{N}^{\mathbb{Q}}(d s, d x) \\
& \quad=\int_{0}^{t} \int_{\mathbb{R}^{*}} \widetilde{N}(d s, d x)-\int_{0}^{t} \int_{\mathbb{R}^{*}} l_{s}(x) \nu(d x) d s,
\end{aligned}
$$

is a $\mathbb{Q}$-local martingale.

Remark 9. We would like to stress that $\sigma_{t}$, the diffusion coefficient, and $N$, the Poisson random measure of process jumps, do not change passing from the original probability $\mathbb{P}$ to the equivalent measure $\mathbb{Q}$, since they are path properties of the process. Heuristically speaking, while process' paths do not change, the Girsanov transformation changes their probability to be realized. 
Taking into consideration Lévy process implies that condition (20) is satisfied provided the existence of two suitable processes $u_{t}$ and $l_{t}$ and this leads to the identification of more than one equivalent martingale measure $\mathbb{Q}$; therefore related markets are incomplete; moreover only particular choices of the processes $u_{t}$ and $l_{t}$ allow for an equivalent measure $\mathbb{Q}$ with a physical meaning. In some particular cases, for example, when the driving process is an exponential Itô-Lévy type process, a meaningful choice is represented by the so-called Esscher transformation which is defined taking $l_{t}=\left(e^{\alpha x}-1\right)$, $\alpha \in \mathbb{R}$, and it has the good physical property of minimizing the relative entropy; see, for example [23].

Considering such a transformation in relation with (25), the resulting density $Z_{T}$, solution of $d Z_{t}=Z_{t_{-}} d L_{t}$, where the process $L_{t}$ satisfies

$$
d L_{t}=u_{t} d W_{t}+\int_{\mathbb{R}^{*}} l_{t}(x) \widetilde{N}(d t, d x),
$$

is called Doléans-Dade exponential or stochastic exponential, and it will be denoted by $Z_{T}=\mathscr{E}(L)_{T}$. In particular if $Z$ evolves according to (25), we have

$$
\begin{aligned}
Z_{T}= & \mathscr{E}(L)_{T}=\exp \left\{L_{t}-\frac{1}{2}\left[L_{t}^{c}, L_{t}^{c}\right]\right\} \prod_{0 \leq s \leq t}\left[1+\Delta L_{s}\right] e^{-\Delta L_{s}} \\
= & \exp \left\{\int_{0}^{T} u_{s} d W_{s}-\frac{1}{2} \int_{0}^{T} u_{s}^{2} d s\right. \\
& \left.\quad-\int_{0}^{T} \int_{\mathbb{R}^{*}} l_{t}(x) \nu(d x) d s\right\} \\
& \times \prod_{0 \leq s \leq T}\left(1+l_{s}\left(\Delta L_{s}\right)\right),
\end{aligned}
$$

where we have denoted by $L_{t}^{c}$ the continuous part of $L_{t}$ with $[\cdot, \cdot]$ being the quadratic variation and denoted by $\Delta Y_{t}:=Y_{t}-$ $Y_{t-}$ the jump occurring at time $t$. We refer to [18], in particular to Sections 5.4.3, 5.4.4, and 5.4.5 and references therein, for further details about both the Esscher transformation and the Doléans-Dade exponentials. Let us note that (see, for example, [18, Chapter 5]) $L_{t}$ is a local martingale since it can be rewritten as a Lévy type stochastic integral thanks to the following result.

Theorem 10. Let one assume that for any $t \geq 0 \sqrt{G_{t}}$ is a predictable process such that

$$
\mathbb{E} \int_{0}^{t} G(s) d s<\infty,
$$

and that $t \geq 0 K(t, x)$ is a predictable process satisfying

$$
\begin{gathered}
\mathbb{E} \int_{0}^{t} \int_{|x|>1}|K(s, x)|^{2} \nu(d x) d s<\infty, \\
\mathbb{P}\left(\int_{0}^{t} \int_{|x|>1}|K(s, x)|^{2} \nu(d x) d s<\infty\right)=1 ;
\end{gathered}
$$

then $L_{t}$ is any Lévy type stochastic integral of the form

$$
\begin{aligned}
d L_{t}= & G_{t} d t+F_{t} d W_{t}+\int_{|x|<1} H(t, x) \widetilde{N}(d t, d x) \\
& +\int_{|x|>1} K(t, x) N(d t, d x) ;
\end{aligned}
$$

then $L_{t}$ is a local martingale if and only if

$$
G_{t}+\int_{|x|>1} K(t, x) \nu(d x)=0, \text { a.s. }
$$

\section{Financial Framework}

In what follows we will use results stated in Section 2 to analyse the problem of pricing and hedging contingent claim written on underlyings subjected to a risk of both diffusive and jump type, hence allowing for underlyings' dynamics with random discontinuities. Our financial framework will be defined as a market composed by two securities: a riskless asset, for example, a bond, a bank account, and so forth, $B=\left\{B_{t}: 0 \leq t \leq T\right\}$, solution to the following deterministic differential equation:

$$
\begin{gathered}
d B_{t}=r_{t} B_{t} d t, \quad 0 \leq t \leq T, \\
B_{0}=1,
\end{gathered}
$$

where $r_{t}$ is the risk-free interest rate, and a risky security (or stock) $S:=\left\{S_{t}: 0 \leq t \leq T\right\}$ solution to the following:

$$
\begin{gathered}
d S_{t}=S_{t^{-}}\left[\mu_{t} d t+\sigma_{t} d W_{t}+\int_{\mathbb{R}^{*}} \gamma_{t}(x) \widetilde{N}(d t, d x)\right], \\
0 \leq t \leq T, \\
S_{0}=s \in \mathbb{R},
\end{gathered}
$$

where $\mu_{t}$ and $\sigma_{t}$ are predictable processes called respectively drift and diffusion, while $\gamma_{t}(x) \in \mathscr{H}_{v}$ is the jump component. We thus have that the stock price $S_{t}$ is a càdlàg process described by a geometric Itô-Lévy process. Thus the investor in the risky asset is exposed to a diffusion risk, caused by the Brownian component, as well as to a jump component risk given by $\int_{\mathbb{R}^{*}} \gamma_{t}(x) \widetilde{N}(d t, d x)$.

In what follows we will denote by $V:=\left\{V_{t}: 0 \leq t \leq T\right\}$ the wealth stochastic process representing the total value of the investor's portfolio at time $t$, given an initial wealth $V_{0}>0$. In particular the investor, at a given time $t$, holds $\pi_{t}$ share of the risky stock, whilst the remaining part of his total wealth, $V_{t}-\pi_{t}$, is invested in the riskless bond. Let us note that, in order to avoid an arbitrage opportunity (see, for example, [24]) we further assume the portfolio to be self-financing; that is, there is no exogenous infusion or withdrawal of money. This implies that the instantaneous variation of the wealth value is caused uniquely by assets' prices variations and not by injecting or withdrawing funds from outside; hence the self-financing condition reads as follows. 
Definition 11 (self-financing strategy). A self-financing strategy is a pair $\left(V_{t}, \pi_{t}\right)$, where $\pi_{t}$ is a predictable process such that

$$
V_{t}=V_{T}+\int_{t}^{T}\left(\left(V_{t}-\pi_{t}\right) \frac{d B_{t}}{B_{t}}+\pi_{t} \frac{d S_{t}}{S_{t}}\right),
$$

with

$$
\begin{gathered}
\mathbb{E}\left[\int_{0}^{T}\left|\sigma_{t} \pi_{t}\right|^{2} d t\right]<+\infty, \\
\mathbb{E}\left[\int_{0}^{T} \pi_{t}^{2} \gamma_{t}^{2}(x) \nu(d x) d t\right]<+\infty .
\end{gathered}
$$

Within the framework defined in Section 3, we have that a contingent claim, with payoff at maturity time $T$ given by $\xi$, is said to be hedgeable if there exists a self-financing strategy $\left(V_{t}, \pi_{t}\right)$ of the form (39) that replicates its payoff, in particular the following result holds.

Proposition 12. A self-financing strategy solves a linear Lipschitz BSDE of the form

$$
\begin{gathered}
-d V_{t}=r_{t} V_{t} d t-\pi_{t} \sigma_{t} u_{t} d t-\left\langle\pi_{t} \gamma_{t}, l_{t}\right\rangle_{\nu} d t+\pi_{t} \sigma_{t} d W_{t} \\
+\int_{\mathbb{R}^{*}} \pi_{t} \gamma_{t}(x) \widetilde{N}(d t, d x), \\
V_{T}=\xi .
\end{gathered}
$$

Proof. Let us consider an asset evolving according to (38) together with a risk-less security as in (37). A straightforward substitution of (38) and (37) into equation for the selffinancing strategy (39) leads to the conclusion that the selffinancing strategy solves the BSDE:

$$
\begin{gathered}
-d V_{t}=r_{t} V_{t} d t+\pi_{t}\left(\mu_{t}-r_{t}\right) d t+\pi_{t} \sigma_{t} d W_{t} \\
+\int_{\mathbb{R}^{*}} \pi_{t} \gamma_{t}(x) \widetilde{N}(d t, d x), \\
V_{T}=\xi .
\end{gathered}
$$

Note that applying the Girsanov Theorem 7 to the stock price (39) with Radon-Nikodym density given as in (32) we have that the martingale condition (20) in Theorem 7 has to hold to have the discounted price $e^{-\int_{0}^{t} r_{s} d s} S_{t}$ to be a martingale under a risk-neautral measure $\mathbb{Q}$ equivalent to the real world measure $\mathbb{P}$; therefore substituting (20) into (42), we obtain that the self-financing strategy $V_{t}$ has to satisfy the following BSDE:

$$
\begin{gathered}
-d V_{t}=r_{t} V_{t} d t-\pi_{t} \sigma_{t} u_{t} d t-\left\langle\pi_{t} \gamma_{t}, l_{t}\right\rangle_{\nu} d t+\pi_{t} \sigma_{t} d W_{t} \\
+\int_{\mathbb{R}^{*}} \pi_{t} \gamma_{t}(x) \widetilde{N}(d t, d x), \\
V_{T}=\xi,
\end{gathered}
$$

which is an equation of the form (15) with

$$
\begin{aligned}
& X_{t}=V_{t}, \quad Y_{t}=-\pi_{t} \sigma_{t}, \quad U_{t}=-\pi_{t} \gamma_{t}(x), \\
& \alpha_{t}=0, \quad \beta_{t}=r_{t}, \quad \delta_{t}=u_{t}, \quad \zeta_{t}=l_{t} .
\end{aligned}
$$

Proposition 12 implies that we can apply Theorem 6 to obtain a formula for pricing a contingent claim in a market consisting of a risky asset driven by a jump-diffusion dynamic; indeed we have the following.

Theorem 13. Let $\left(V_{t}, \pi_{t} \sigma_{t}, \pi_{t} \gamma_{t}\right)$ be the solution in $S \times \mathscr{H} \times \mathscr{H}_{v}$ of the following linear BSDE:

$$
\begin{gathered}
-d V_{t}=r_{t} V_{t} d t-\pi_{t} \sigma_{t} u_{t} d t-\left\langle\pi_{t} \gamma_{t}, l_{t}\right\rangle_{\nu} d t+\pi_{t} \sigma_{t} d W_{t} \\
+\int_{\mathbb{R}^{*}} \pi_{t} \gamma_{t}(x) \widetilde{N}(d t, d x), \\
V_{T}=\xi,
\end{gathered}
$$

so that the assumptions of Theorem 5 are satisfied. Let one further suppose that the assumptions of the Girsanov Theorem 7 hold, so that there exists an equivalent measure $\mathbb{Q}$ with RadonNikodym density $Z$ as in (32); then a solution of the BSDE (45) is given by

$$
\begin{array}{r}
V_{t}=\mathbb{E}\left[e^{-\int_{t}^{T} r_{s} d s} Z_{t} \xi \mid \mathscr{F}_{t}\right]=\mathbb{E}_{\mathbb{Q}}\left[e^{-\int_{t}^{T} r_{s} d s} \xi \mid \mathscr{F}_{t}\right], \\
0 \leq t \leq T \text { a.s. }
\end{array}
$$

Proof. The proof is a straightforward application of Theorem 6 to the particular case of a BSDE of the form (45).

From now on we will deal mainly with a BSDE of the form of (41).

3.1. The Black-Scholes Model. Let us consider the standard Black-Scholes (BS) model, where the driving process is a diffusion without jumps; see, for example, $[25,26]$ for details; hence the riskless bond $B_{t}$ solves

$$
\begin{gathered}
d B_{t}=r B_{t} d t, \quad 0 \leq t \leq T, \\
B_{0}=1,
\end{gathered}
$$

while the risky asset $S_{t}$ is the solution of

$$
\begin{gathered}
d S_{t}=\mu S_{t} d t+\sigma S_{t} d W_{t}, \quad 0 \leq t \leq T, \\
S_{0}=s
\end{gathered}
$$

for some constant parameters $r, \mu$, and $\sigma$. It is well known that the BS model describes a complete and free of arbitrage market; hence there exists a unique equivalent risk-neutral measure $\mathbb{Q}$, and $e^{-r t} S_{t}$ is a $\mathbb{Q}$-martingale. Applying Girsanov Theorem 7 to the Brownian case with $u_{t}=(r-\mu) / \sigma$, we have that under the new measure $\mathbb{Q}:=Z_{T} \mathbb{P}$, with

$$
Z_{T}=\exp \left\{\int_{0}^{T} \frac{r-\mu}{\sigma} d W_{s}-\frac{1}{2} \int_{0}^{T}\left(\frac{r-\mu}{\sigma}\right)^{2} d s\right\},
$$

the stock price evolves according to

$$
d S_{t}=r S_{t} d t+\sigma S_{t} d W_{t}^{\mathbb{Q}}, \quad 0 \leq t \leq T .
$$


Suppose that we want to hedge a call option $\xi$ written on an underlying whose dynamic is described by (48), with payoff given by $\Phi\left(S_{T}\right)=\max \left(0, S_{T}-K\right)=:\left(S_{T}-K\right)_{+}$, where $K$ is the so-called strike price; then we have to construct a replicating portfolio according to Proposition 12, without jump component. The latter implies that we have to solve the following BSDE:

$$
V_{t}=\left(S_{T}-K\right)_{+}-\int_{t}^{T}\left(r V_{s}+\pi_{s} \sigma u_{s}\right) d s-\int_{t}^{T} \pi_{s} \sigma d W_{s}
$$

By Theorem 6 together with Girsanov Theorem 7 we have that the initial value of the replicating portfolio, which coincides with the fair price of the claim, is given by

$$
\begin{aligned}
X_{0} & =\mathbb{E}_{\mathbb{Q}}\left[e^{-r T}\left(S_{T}-K\right)_{+}\right] \\
& =K e^{-r T} \Phi\left(-d\left(0, S_{0}-\sigma \sqrt{T}\right)\right)-S_{0} \Phi\left(-d\left(0, S_{0}\right)\right),
\end{aligned}
$$

a result obtained exploiting the fact that, for any given $t \epsilon$ $[0, T]$, the random variables $\ln S_{t}$ are normally distributed; therefore we explicitly know their density functions, $\Phi$ being the corresponding cumulative distribution function and

$$
d\left(t, S_{t}\right):=\frac{\ln \left(S_{t} / K\right)+\left(r+\left(\sigma^{2} / 2\right)\right)(T-t)}{\sigma \sqrt{T-t}} .
$$

It can be easily checked that the fair price given in (52) coincides with the fair price obtained via standard methods; see, for example, [26, Chapter 5].

3.2. Local Volatility Models. A first generalization of the model proposed in Section 3.1 is realized by the so-called local volatility models (LVM), where both the drift and the volatility parameters are no longer constant. Empirical evidences have shown that latter models, which in general do not have explicit solutions, fit better real data when compared to the standard BS setting, in particular with respect to the analysis of the implied volatility surface; see, for example [27, 28]. Let us then consider a riskless bond $B_{t}$ solution to

$$
\begin{gathered}
d B_{t}=r_{t} B_{t} d t, \quad 0 \leq t \leq T, \\
B_{0}=1,
\end{gathered}
$$

and a risky asset $S_{t}$

$$
\begin{gathered}
d S_{t}=\mu\left(S_{t}\right) d t+\sigma\left(S_{t}\right) d W_{t}, \quad 0 \leq t \leq T, \\
S_{0}=s,
\end{gathered}
$$

where $r_{t}, \mu$, and $\sigma$ are $\mathscr{F}$-predictable processes satisfying standard Lipschitz continuity and linear growth assumptions, so that there exists a unique solution to (48); see, for example, [29]. Within this framework one of the most used models is the constant elasticity of variance $(\mathrm{CEV})$ model, where the volatility term is of the form $S_{t}^{\beta / 2}$, for $0 \leq \beta \leq 2$. Again applying the Girsanov theorem, with $u_{t}=\left(r_{t}-\mu\left(S_{t}\right)\right) / \sigma\left(S_{t}\right)$, the stock price under the risk-neutral measure $\mathbb{Q}$ evolves according to

$$
d S_{t}=r_{t} d t+\sigma\left(S_{t}\right) d W_{t}^{\mathbb{Q}}, \quad 0 \leq t \leq T .
$$

If we want to construct a replicating portfolio to hedge a contingent claim $\xi$, with terminal payoff given by $\xi=$ $\left(S_{T}-K\right)_{+}$, then the portfolio dynamic has to solve the following BSDE:

$$
\begin{aligned}
V_{t}= & \left(S_{T}-K\right)_{+}-\int_{t}^{T}\left(r_{s} V_{s}+\pi_{s} \sigma\left(S_{s}\right) u_{s}\right) d s \\
& -\int_{t}^{T} \pi_{s} \sigma\left(S_{s}\right) d W_{s} ;
\end{aligned}
$$

hence, by Theorem 13 and (46), we have that the initial value of the replicating portfolio is given by

$$
X_{0}=\mathbb{E}_{\mathbb{Q}}\left[e^{-r T}\left(S_{T}-K\right)_{+}\right] .
$$

Note that, as mentioned before, an analytic solution to (58), namely, an explicit solution for the fair price problem, does not exist; nevertheless effective numerical approximations can be given; see, for example, [8, Section 12$]$.

3.3. The Black-Scholes Model with Jumps. A different generalization of the B-S model introduced in Section 3.1 is obtained considering a stock price driven by a general Lévy process instead of a standard Brownian motion. Latter generalization is motivated by empirical results (see, for example, [8]) which show the presence of leaps in the evolution of real stock markets' quantities; hence we are in presence of discontinuities that cannot be modelled using a Brownian type approach since the Brownian paths are continuous. Latter analysis implies the need for a more general type of random processes to be used, hence allowing for jumps in the stocks' prices dynamics. Therefore a natural improvement is to consider Lévy type drivers. Nevertheless it is crucial to underline that even if Lévy processes fit better to empirical data than the Brownian counterpart does, they are mathematically more difficult to treat. In fact not only is an analytical solution to the pricing equation no longer available, but also the market is not anymore complete. Let us then consider a riskless bond $B_{t}$ solution to

$$
\begin{gathered}
d B_{t}=r B_{t} d t, \quad 0 \leq t \leq T, \\
B_{0}=1
\end{gathered}
$$

and a risky asset $S_{t}$

$$
\begin{gathered}
d S_{t}=\mu S_{t} d t+\sigma S_{t} d W_{t}+\int_{\mathbb{R}^{*}} S_{t_{-}} x \widetilde{N}(d t, d x), \quad 0 \leq t \leq T, \\
S_{0}=s,
\end{gathered}
$$

where the Poisson component has Lévy measure $v$. The solution to (60) can be found exploiting the stochastic exponential introduced in (32); in particular we have

$$
S_{t}=s e^{\left(\mu-\left(\sigma^{2} / 2\right)\right) t+\sigma W_{t}} \prod_{i=1}^{N_{t}}\left(1+Z_{i}\right)
$$


where $N_{t}$ is a Poisson process independent of the Brownian motion $W_{t}$. Since we are now dealing with a Lévy process, a unique equivalent measure $\mathbb{Q}$ does not exist; see, for example, [18]; namely, it is not possible to uniquely determine the solution couple $\left(u_{t}, l_{t}\right)$. Nevertheless, in the case of a geometric Lévy process, a suitable choice can be obtained by the so-called Esscher transform and the density $Z_{T}$ takes the form of a Doléans-Dade exponential (32).

In particular if we define $u_{t}:=(r-\mu) / \sigma$ and $l_{t}=e^{x}-1$ (see Section 2.2) we have that the asset has mean return $r$ under the risk-neutral measure $\mathbb{Q}$; hence the discounted price is a $\mathbb{Q}$-martingale; namely, it satisfies the following equation:

$$
\begin{gathered}
d S_{t}=r S_{t} d t+\sigma S_{t} d W_{t}^{\mathbb{Q}}+\int_{\mathbb{R}^{*}} S_{t_{-}} x \widetilde{N}^{\mathbb{Q}}(d t, d x), \quad 0 \leq t \leq T, \\
S_{0}=s ;
\end{gathered}
$$

moreover hedging the contingent claim with terminal payoff given by $\xi=\left(S_{T}-K\right)_{+}$, we have that the portfolio satisfies the following BSDE:

$$
\begin{aligned}
V_{t}= & \left(S_{T}-K\right)_{+}-\int_{t}^{T}\left(r V_{s}+\pi_{s} \sigma u_{s}+\left\langle l_{s}, \pi_{s} x\right\rangle_{\nu}\right) d s \\
& +\int_{t}^{T} \pi_{s} \sigma d W_{s}+\int_{t}^{T} \int_{\mathbb{R}^{*}} \pi_{s} x \widetilde{N}(d s, d x),
\end{aligned}
$$

that is, a linear BSDE of the form (43); hence, by Theorem 13, we have that the value $V_{0}$ of the portfolio at initial time is given by

$$
X_{0}=\mathbb{E}_{\mathbb{Q}}\left[e^{-r T}\left(S_{T}-K\right)_{+}\right]
$$

3.4. Life Insurance Portfolio. Backward stochastic differential equations have a wide range of applications, from standard examples, as stated in Sections 3.1, 3.2, and 3.3, to less typical scenario as the ones characterizing actuarial applications; see, for example [2] for details. In what follows we solve a novel life insurance pricing problem. Let us assume that we are dealing with the B-S financial model described in Section 3.1; namely, we have a riskless bond $B_{t}$ which evolves according to

$$
\begin{gathered}
d B_{t}=r B_{t} d t, \quad 0 \leq t \leq T, \\
B_{0}=1,
\end{gathered}
$$

and a risky asset $S_{t}$ satisfying

$$
\begin{gathered}
d S_{t}=\mu S_{t} d t+\sigma S_{t} d W_{t}^{1}, \quad 0 \leq t \leq T \\
S_{0}=s .
\end{gathered}
$$

Let us consider a stochastic mortality intensity $J:=\left\{J_{t}\right\}_{t \in[0, T]}$, $T<+\infty$, which is modelled by the following SDE:

$$
\begin{gathered}
d J_{t}=\mu^{J}\left(J_{t}\right) d t+\sigma^{J}\left(J_{t}\right) d W_{t}^{2}, \\
J_{0}=j,
\end{gathered}
$$

where the process $J$ is independent of the financial market described by the couple $(B, S)$. Since we aim at considering a life insurance liability problem, then we will analyse the dynamics of life insurance equity-linked claims. The uncertainty in this type of investments comes mainly from the fact that we cannot know how mortality rates will evolve.

Let us now assume that we are willing to construct a portfolio consisting of an arbitrary, but finite, number $n$ policies, each of which relates to a unique insured person whose lifetime is modelled via $n$ inhomogeneous Poisson process $\tau_{i}, i=1, \ldots, n$; that is,

$$
\mathbb{P}\left(\tau_{i}>t\right)=e^{\int_{0}^{t} \lambda(s) d s}, \quad i=1, \ldots, n,
$$

where $\lambda:[0, T] \rightarrow \mathbb{R}_{+}$is a deterministic function representing the mortality intensity; then the insurance payment process $P:=\left\{P_{t}\right\}_{t \in[0, T]}$ can be modelled as follows:

$$
\begin{array}{r}
P_{t}=\int_{0}^{t}\left(n-N_{s}\right) H_{s} d s+\int_{0}^{t} G_{s} d N_{s}+\left(n-N_{T}\right) \xi \mathbb{1}_{\{t=T\}}, \\
0 \leq t \leq T,
\end{array}
$$

where the claims $H, G$, and $\xi:[0, T] \times \mathbb{R}_{+} \rightarrow \mathbb{R}_{+}$ are measurable functions; $\mathbb{1}_{\{t=T\}}$ stands for the characteristic function of the set $\{t=T\}$, while $N$ is a counting measure representing a death counting process for a life insurance portfolio with $n$ policies linked to $n$ different insured persons. A classical credit default swap is modelled setting $n=1$ and $F=0$, while a collective credit risk setting is obtained taking $\xi=H=0$ and $G=g(t) \mathbb{1}_{\{t=T\}}$ so that it represents the credit loss in case of default; see, for example, [2] and references therein for more details. Heuristically the process $P$ can be seen as a stream of liabilities since it is defined by a continuous payments stream $H$, a random payment process $G$, which is financially defined as the death benefits process, and a claim $\xi$, the so-called survival benefit, which pays off at maturity time. Latter defined claims, namely, $H, \xi$, and $G$, depend upon the financial market defined by (65) and (66). During last years growing attention has been attracted by the so-called mortality derivatives. Such type of contracts are mainly stipulated in order to hedge mortality risk. We will show that the BSDE approach introduced in Section 2 can be usefully exploited to explicitly find a strategy which replicates a mortality bond by mean of some specific investments.

Let us assume that the mortality bond is already priced; hence we already are within the risk-neutral framework with respect to a certain risk-neutral measure $\mathbb{Q}$; therefore, taking into account definitions given in Section 2.2, the density $Z$ is given as in (32) and the processes $u_{t}$ and $l_{t}$ are as in Section 3.3. Our goal is to solve a hedging problem; namely, we aim at finding a replicating strategy for the insurance payment process (69). Exploiting Proposition 12, 
we obtain that the replicating portfolio evolves according to the following BSDE:

$$
\begin{aligned}
V_{t}= & \left(n-J_{T}\right) \xi+\int_{t}^{T}\left(-r V_{s}-u_{s} Z_{s}\right)+\lambda(s)\left(n-N_{s_{-}}\right) Z_{s} l_{s} \\
& +H_{s}\left(n-N_{s_{-}}\right)+\lambda(s) G_{s}\left(1+l_{s}\right)\left(n-N_{s_{-}}\right) d s \\
& -\int_{t}^{T} Z_{s} d W_{s}-\int_{t}^{T} U_{s} \widetilde{N}(d s), \quad 0 \leq t \leq T,
\end{aligned}
$$

and we are in position to apply Theorem 13 to find that a solution of (70) is given by

$$
V_{t}=\mathbb{E}^{\mathbb{Q}}\left[\int_{t}^{T} e^{-r(s-t)} d P_{s} \mid \mathscr{F}_{t}\right] .
$$

Proposition 14. In a market such that the unsystematic mortality risk can be diversified, a solution $\left(V_{t}, Y_{t}, Z_{t}\right) \in S \times$ $\mathscr{H} \times \mathscr{H}_{\nu}$ to the BSDE

$$
\begin{aligned}
V_{t}=\xi & +\int_{t}^{T}\left(-r V_{s}-u_{s} Z_{s}\right)+\lambda(s)\left(n-N_{s_{-}} Z_{s} l_{s}\right) \\
& +H_{s}\left(n-N_{s_{-}}\right)+\lambda(s) G_{s}\left(1+l_{s}\right)\left(n-N_{s_{-}}\right) d s \\
& -\int_{t}^{T} Y_{s} d W_{s}-\int_{t}^{T} Z_{s} \widetilde{N}(d s), \quad 0 \leq t \leq T,
\end{aligned}
$$

when the claim to hedge is given by $\xi=\left(n-N_{T}\right)\left(S_{T}-K\right)_{+}$and the mortality rate is constant, is given by

$$
\begin{gathered}
V_{t}=\left(n-N_{t}\right) e^{-\lambda(T-t)}\left[e^{-r(T-t)} \Phi\left(d\left(t, S_{t}\right)-\sigma \sqrt{T-t}\right)\right. \\
\left.-S_{t} \Phi\left(-d\left(t, S_{t}\right)\right)\right], \\
Y_{t}=\left(n-N_{t}\right) e^{-\lambda(T-t)} \sigma S_{t} \Phi\left(-d\left(t, S_{t}\right)\right), \\
Z_{t}=e^{-\lambda(T-t)}\left[K e^{-r(T-t)} \Phi\left(d\left(t, S_{t}\right)-\sigma \sqrt{T-t}\right)\right. \\
\left.-S_{t} \Phi\left(-d\left(t, S_{t}\right)\right)\right],
\end{gathered}
$$

where $\Phi$ is the cumulative distribution of the standard Gaussian random variable and $d$ is given as in (53).

Proof. We are interested in hedging the claim $\xi=(n-$ $\left.N_{T}\right)\left(S_{T}-K\right)_{+}$, which is a call option, with strike price $K$, written on a given fund (see Section 3.1) which collects $n$ insured persons. In the present case $H=G=0$, moreover since we have assumed that the market believes that the unsystematic mortality risk can be diversified, then $l_{t}=0$; see, for example, [2, Section 9.4], for details. It follows that the replicating portfolio for the claim $\xi$ (see (70)) turns out to be described by the following BSDE:

$$
\begin{aligned}
V_{t}= & \xi+\int_{t}^{T}\left(-r V_{s}-u_{s} Z_{s}\right) d s-\int_{t}^{T} Y_{s} d W_{s} \\
& -\int_{t}^{T} Z_{s} \widetilde{N}(d s), \quad 0 \leq t \leq T,
\end{aligned}
$$

or equivalently, under the risk-neutral measure $\mathbb{Q}$, by the following:

$$
\begin{array}{r}
V_{t}=\xi-\int_{t}^{T} r V_{s} d s-\int_{t}^{T} Y_{s} d W_{s}^{\mathbb{Q}}-\int_{t}^{T} Z_{s} \widetilde{N}^{\mathbb{Q}}(d s), \\
0 \leq t \leq T,
\end{array}
$$

which is an equation of the form (41) and since assumptions of Theorem 5 are satisfied, it admits a unique solution. Moreover, exploiting Theorem 13, we can compute such a solution $V_{t}$ from the standard B-S model stated in Section 3.1. In particular by the B-S pricing formula (51), the BS price denoted by $P_{B S}$ reads as follows:

$$
V_{t}=\mathbb{E}\left[e^{-\lambda(T-t)} \xi \mid \mathscr{F}_{t}\right]=\left(n-N_{t}\right) e^{-\lambda(T-t)} P_{B S} ;
$$

therefore a solution to (75) is given by

$$
\begin{gathered}
V_{t}=\left(n-N_{t}\right) e^{-\lambda(T-t)}\left[K e^{-r(T-t)} \Phi\left(d\left(t, S_{t}\right)-\sigma \sqrt{T-t}\right)\right. \\
\left.-S_{t} \Phi\left(-d\left(t, S_{t}\right)\right)\right], \\
Y_{t}=\left(n-N_{t}\right) e^{-\lambda(T-t)} \sigma S_{t} \Phi\left(-d\left(t, S_{t}\right)\right), \\
Z_{t}=e^{-\lambda(T-t)}\left[K e^{-r(T-t)} \Phi\left(d\left(t, S_{t}\right)-\sigma \sqrt{T-t}\right)\right. \\
\left.-S_{t} \Phi\left(-d\left(t, S_{t}\right)\right)\right] .
\end{gathered}
$$

In particular the fair price of the claim $\xi$, regardless of the initial wealth $V_{0}$ of the portfolio, is given by

$$
\begin{gathered}
V_{0}=n e^{-\lambda T}\left[K e^{-r T} \Phi\left(d\left(0, S_{0}\right)-\sigma \sqrt{T}\right)\right. \\
\left.-S_{0} \Phi\left(-d\left(0, S_{0}\right)\right)\right] .
\end{gathered}
$$

\section{Conclusion}

The theory of backward stochastic differential equations has been characterized by an impressive growth of interest from the applicative point of view under the pushing of a wide variety of different scientific communities, spanning from pure mathematicians to engineers and from biologists to risk management's practitioners. A key ingredient behind such an increasing interest relies in the flexibility of the BSDEs approach which can be further improved allowing to include a rather general type of stochastic perturbations.

In the present paper we have showed how BSDEs setting (see Section 2) and related results (see, for example, the predictable representation theorem, Theorem 13 , and the Girsanov theorem, Theorem 7) can be fruitfully exploited to obtain concrete results in a wide set of problems which arise in modern theory of quantitative finance, with a focus on those related to option pricing/hedging (see Section 3). In particular, after having recalled standard results about classical Brownian framework (see, for example, Section 3.1) 
we moved to the more recent settings characterized by the BSDEs' approach with perturbations of Lévy type (see Section 2) whose related techniques are then exploited (see Section 3.4) to develop an innovative model focusing our attention on a less standard life insurance scenario. We would like to note that, despite the fact that the mathematical literature about such kind of problems is not so developed, insurance contracts actually constitute a large market share within the set of all the traded contract nowadays. Let us note that even if the generalizations of the Black-Scholes model to nonconstant volatility models plus jump perturbations (see Sections 3.2 and 3.3) better fit empirical time series, they miss analytical solutions: therefore numerical evaluation is necessary; see, for example $[8,18]$.

We would like to underline that BSDEs' field of potential applications is much wider than it has been stated in the present paper. As examples there are recent applications concerning the so-called dynamic risk measures which are based upon nonlinear expectations ( $g$-expectations) developed by Peng (see, for example [2, 30, 31]) BSDEs with time-delayed generator (see, for example, $[2,32]$ ) where the problem of a big investor who can influence the market prices is treated, and BSDE with reflecting barriers (see, for example, [2]) also in connection with applications to exotic American options pricing and portfolio with consumption problems (see, for example, [33]).

\section{Conflict of Interests}

The authors declare that they have no conflict of interests regarding the publication of this paper.

\section{Acknowledgment}

The authors would like to thank the anonymous referee for his comments and suggestions.

\section{References}

[1] S. Crépey, Financial Modeling. A Backward Stochastic Differential Equations Perspective, Springer Finance Textbooks, Springer, Heidelberg, Germany, 2013.

[2] L. Delong, Backward Stochastic Differential Equations with Jumps and Their Actuarial and Financial Applications, EAA Series, Springer, 2013.

[3] N. El Karoui, S. Peng, and M. C. Quenez, "Backward stochastic differential equations in finance," Mathematical Finance, vol. 7, no. 1, pp. 1-71, 1997.

[4] E. Pardoux and S. G. Peng, "Adapted solution of a backward stochastic differential equation," Systems \& Control Letters, vol. 14, no. 1, pp. 55-61, 1990.

[5] E. Pardoux and S. Peng, "Backward doubly stochastic differential equations and systems of quasilinear SPDEs," Probability Theory and Related Fields, vol. 98, no. 2, pp. 209-227, 1994.

[6] C. W. Gardiner, Handbook of Stochastic Methods: For Physics Chemistry and the Natural Sciences, Springer, New York, NY, USA, 2009.
[7] R. Situ, Theory of Stochastic Differential Equations with Jumps and Applications, Mathematical and Analytical Techniques with Applications to Engineering, Springer, 2005.

[8] R. Cont and P. Tankov, Financial Modelling with Jump Processes, Chapman \& Hall, CRC Press, 2004.

[9] W. Schoutens, Lévy Processes in Finance: Pricing Financial Derivates, John Wiley \& Sons, New York, NY, USA, 2003.

[10] R. Cont, P. Tankov, and E. Voltchkova, "Hedging with options in models with jumps," in Stochastic Analysis and Applications, vol. 2 of Abel Symposia, pp. 197-217, Springer, Berlin, Germany, 2007.

[11] D. Brigo and F. Mercurio, Interest Rat e Models: Theory and Practice: With Smile, Inflation and Credit, Springer Finance, Springer, New York, NY, USA, 2006.

[12] G. di Nunno and B. Öksendal, Advanced Mathematical Methods for Finance, Springer, Berlin, Germany, 2011.

[13] S. Rong, "On solutions of backward stochastic differential equations with jumps and applications," Stochastic Processes and Their Applications, vol. 66, no. 2, pp. 209-236, 1997.

[14] Y. Ouknine, "Reflected backward stochastic differential equations with jumps," Stochastics and Stochastics Reports, vol. 65, no. 1-2, pp. 111-125, 1998.

[15] D. Nualart and W. Schoutens, "Backward stochastic differential equations and Feynman-Kac formula for Lévy processes, with applications in finance," Bernoulli, vol. 7, no. 5, pp. 761-776, 2001.

[16] S. J. Tang and X. J. Li, "Necessary conditions for optimal control of stochastic systems with random jumps," SIAM Journal on Control and Optimization, vol. 32, no. 5, pp. 1447-1475, 1994.

[17] M.-C. Quenez and A. Sulem, "BSDEs with jumps, optimization and applications to dynamic risk measures," Stochastic Processes and Their Applications, vol. 123, no. 8, pp. 3328-3357, 2013.

[18] D. Applebaum, Lévy Processes and Stochastic Calculus, Cambridge University Press, Cambridge, UK, 2004.

[19] T. Bjork, Y. Kabanov, and W. Runggaldier, "Bond market structure in the presence of marked point processes," Mathematical Finance, vol. 7, no. 2, pp. 211-239, 1997.

[20] B. Öksendal and A. Sulem, "Risk indifference pricing in jump diffusion markets," Mathematical Finance, vol. 19, no. 4, pp. 619637, 2009.

[21] B. Öksendal and A. Sulem, Applied Stochastic Control of Jump Diffusions, Springer, 2005.

[22] I. V. Girsanov, "On transforming a class of stochastic processes by absolutely continuous substitution of measures," Theory of Probability and its Applications, vol. 5, pp. 314-330, 1960.

[23] T. Chan, "Pricing contingent claims on stocks driven by Lévy processes," The Annals of Applied Probability, vol. 9, no. 2, pp. 504-528, 1999.

[24] T. Björk, Arbitrage Theory in Continuous Time, Oxford University Press, 3rd edition, 2009.

[25] F. Black and M. Scholes, "The pricing of option s and corporate liabilities," Journal of Political Economy, vol. 81, pp. 637-654, 1973.

[26] S. E. Shreve, Stochastic Calculus for Finance II, Springer, 2004.

[27] J.-P. Fouque, G. Papanicolaou, and R. Sircar, Derivatives in Financial Markets with Stochastic Volatility, Cambridge University Press, Cambridge, UK, 2000.

[28] J.-P. Fouque, G. Papanicolaou, R. Sircar, and K. Sølna, Multiscale Stochastic Volatility for Equity, Interest Rate, and Credit Derivatives, Cambridge University Press, Cambridge, UK, 2011. 
[29] I. Karatzas and S. Shreve, Brownian Motion and Stochastic Calculus, vol. 113 of Graduate Texts in Mathematics, Springer, New York, NY, USA, 2nd edition, 1991.

[30] L. Di Persio and E. Scandola, "Backward stochastic differential equations driven by Lévy noise with applications in Finance," Interdisciplinary Studies of Complex Systems, no. 3, 2013.

[31] S. Peng, "Backward SDE and related g-expectations," in Backward Stochastic Differential Equations, N. El Karoui and L. Mazliak, Eds., vol. 364 of Pitman Research Notes in Mathematics Series, Longman, Harlow, UK, 1997.

[32] L. Delong and P. Imkeller, "Backward stochastic differential equations with time delayed generators-results and counterexamples," The Annals of Applied Probability, vol. 20, no. 4, pp. 1512-1536, 2010.

[33] F. Cordoni, L. Di Persio, L. Maticiuc, and A. Zalinescu, "A stochastic approach for functional differential equations using BSDE with time-delayed generators, work in progress," work in progress, 2014. 


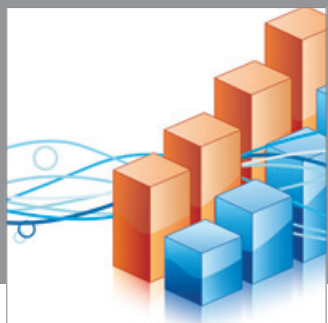

Advances in

Operations Research

mansans

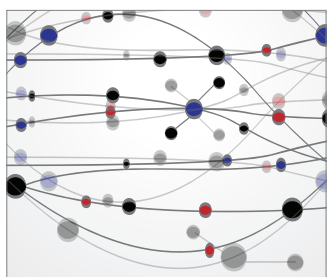

The Scientific World Journal
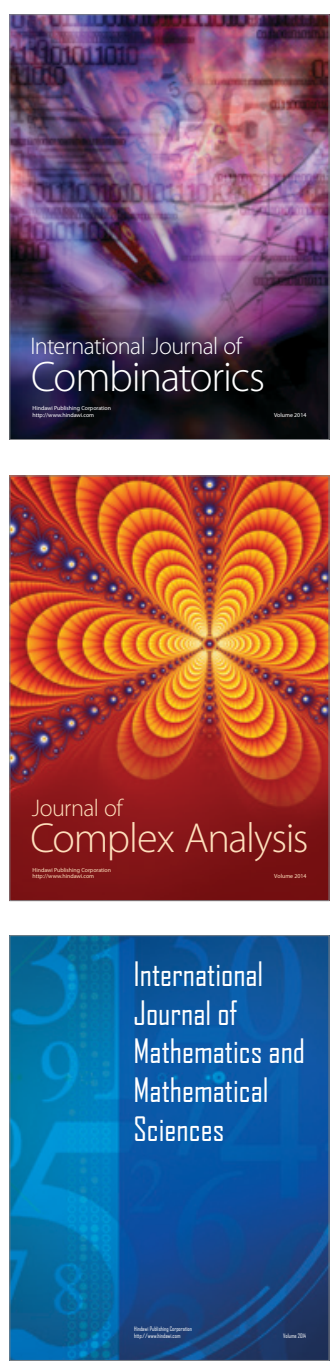
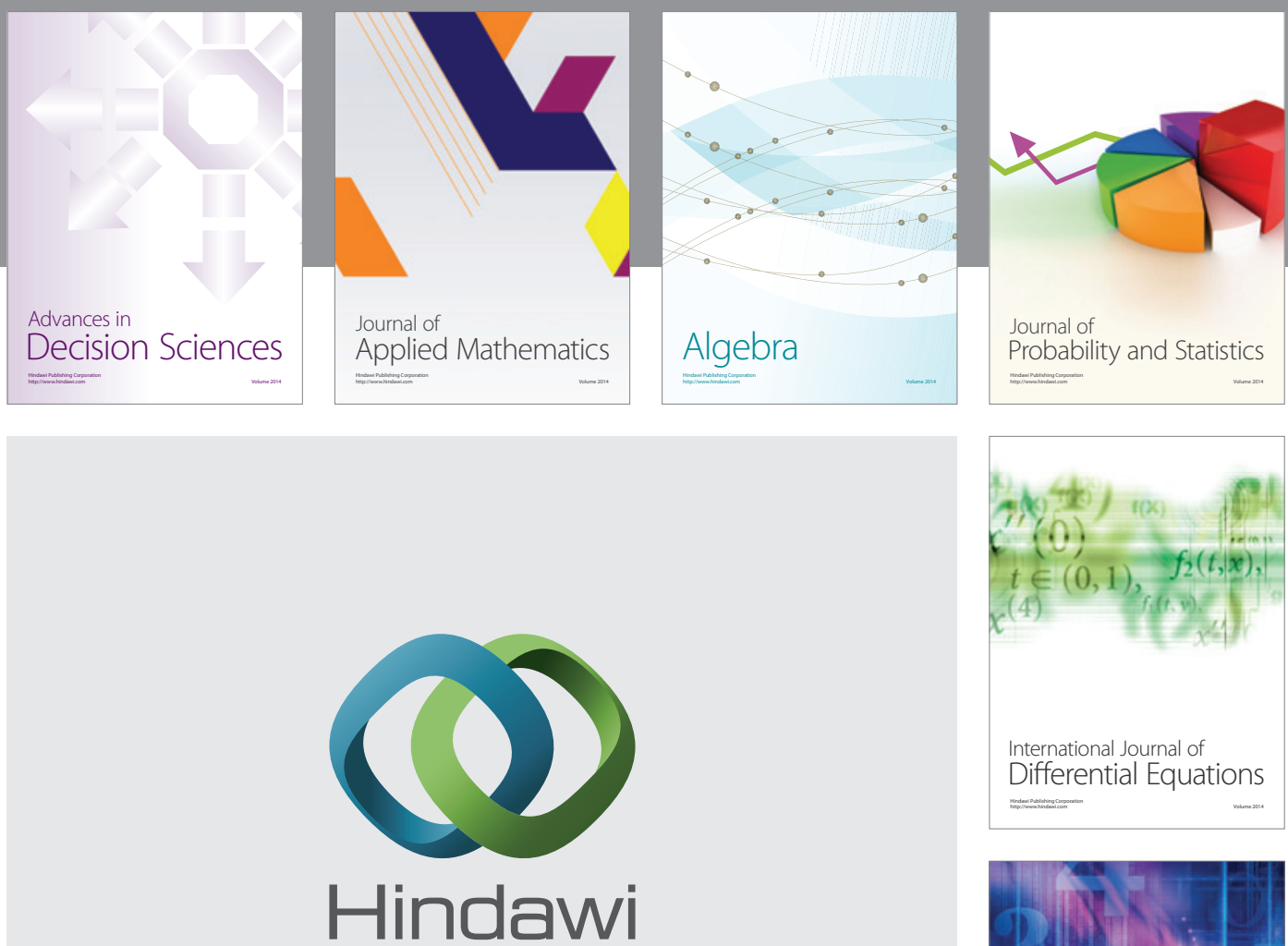

Submit your manuscripts at http://www.hindawi.com
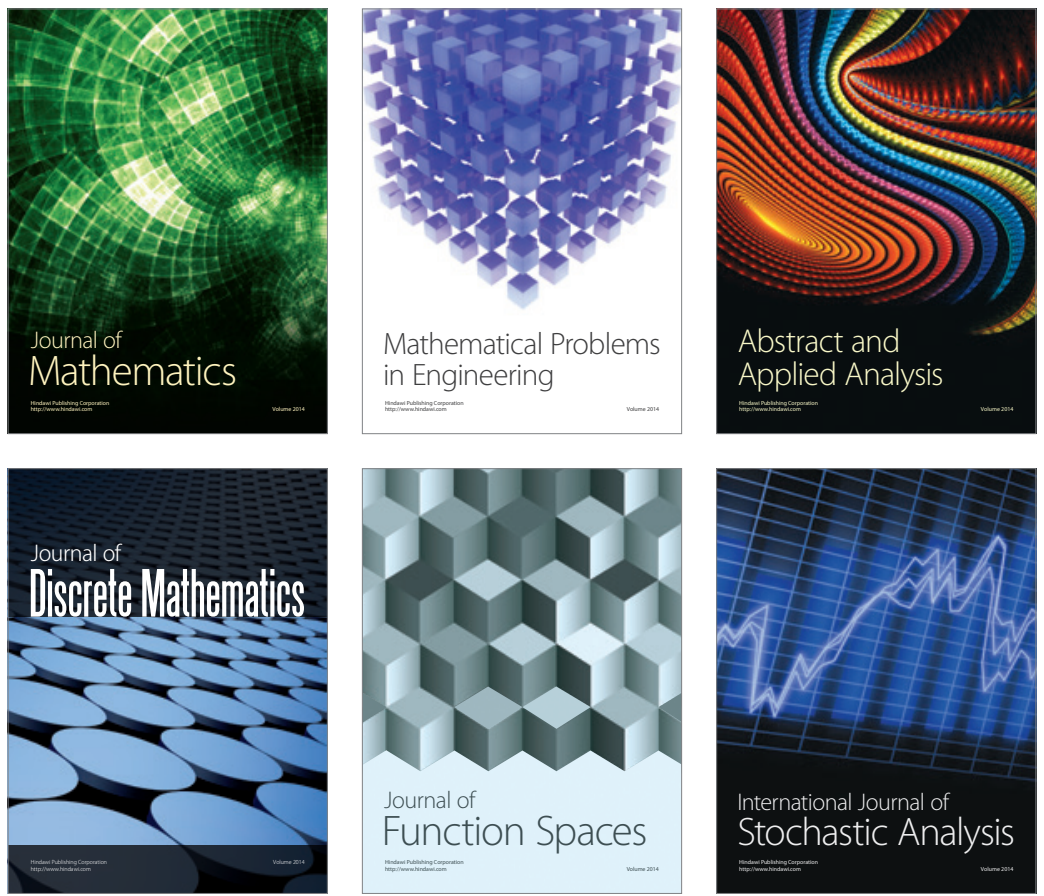

Journal of

Function Spaces

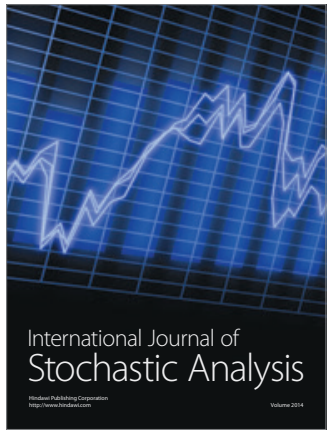

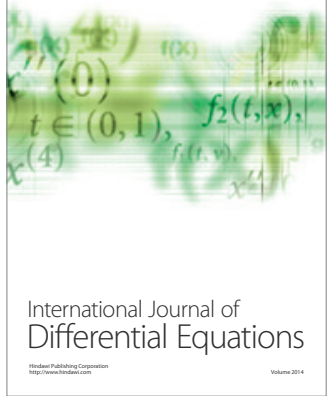
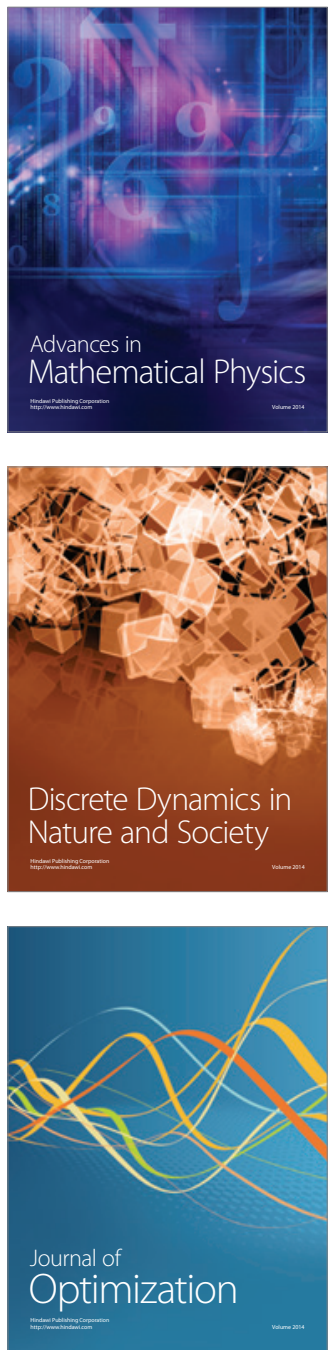